\title{
A Case of Metastatic Melanoma in the Liver Mimicking Hepatocellular Carcinoma
}

\author{
Jae-Kyoung So", Ji-Yun Hong ${ }^{*}$, Min-Woo Chung, Sung-Bum Cho \\ Department of Internal Medicine, Chonnam National University Medical School, Gwangju, Korea
}

Received Dec. 29, 2020

Revised Feb. 24, 2021

Accepted Feb. 26, 2021
The liver is one of the most common sites of metastasis. Although most metastatic liver cancers are hypovascular, some hypervascular metastases, such as those from melanoma, need to be differentiated from hepatocellular carcinoma (HCC) because they may show similar radiologic findings due to their hypervascularity. We encountered a case of multinodular liver masses with hyperenhancement during the arterial phase and washout during the portal venous and delayed phases, which were consistent with imaging hallmarks of HCC. The patient had a history of malignant melanoma and had undergone curative resection 11 years earlier. We performed a liver biopsy for pathologic confirmation, which revealed a metastatic melanoma of the liver. Metastatic liver cancer should be considered if a patient without chronic liver disease has a history of other primary malignancies, and caution should be exercised with hypervascular cancers that may mimic HCC. (J Liver Cancer 2021;21:92-96)

Keywords: Metastasis; Melanoma; Liver neoplasms; Carcinoma, Hepatocellular

\section{INTRODUCTION}

Hepatocellular carcinoma (HCC), the most common primary malignant tumor of the liver, can be diagnosed based on typical imaging hallmarks in high-risk patients with chronic liver disease. The radiologic hallmarks for the diagnosis of HCC are arterial phase hyperenhancement and washout during the portal venous, delayed, or hepatobiliary phase on multiphase computed tomography (CT) or mag-

\section{Corresponding author: Sung-Bum Cho}

Department of Internal Medicine, Chonnam National University Medical School, 42 Jebong-ro, Dong-gu, Gwangju 61469, Korea

Tel. +82-62-220-6296, Fax. +82-62-225-8578

E-mail; portalvein@naver.com

https://orcid.org/0000-0001-9816-3446

* Jae-Kyoung So and Ji-Yun Hong equally contributed to this work as first co-authors. netic resonance imaging (MRI) with extracellular or hepatobiliary contrast agents. ${ }^{1}$ However, some hypervascular metastatic liver tumors may show a similar enhancement pattern and should be differentiated from $\mathrm{HCC} \cdot{ }^{2-4}$ Here, we describe a case of metastatic melanoma of the liver mimicking HCC in a patient with a history of malignant melanoma that had lasted for 11 years.

\section{CASE REPORT}

\section{Clinical findings}

A 65-year-old woman presented with a multinodular liver mass incidentally detected during screening ultrasonography. She had been diagnosed with stage IIA malignant melanoma of the left neck 11 years earlier. After wide excision and the administration of adjuvant interferon-alpha therapy, the pa- 
tient maintained complete remission during the following 5 years. She did not smoke or drink alcohol. She had no associated symptoms, and there were no significant findings during physical examination. The laboratory data revealed a white blood cell (WBC) count of $8,000 / \mu \mathrm{L}$, hemoglobin of $13.4 \mathrm{~g} / \mathrm{dL}$, platelet of $220,000 / \mu \mathrm{L}$, total bilirubin of $0.97 \mathrm{mg} /$ $\mathrm{dL}$, albumin of $4.8 \mathrm{~g} / \mathrm{dL}$, prothrombin time international normalized ratio (PT INR) of 0.94 , aspartate aminotransferase (AST) of $28 \mathrm{IU} / \mathrm{L}$, alanine aminotransferase (ALT) of $21 \mathrm{IU} / \mathrm{L}$, and lactate dehydrogenase (LDH) of $688 \mathrm{IU} / \mathrm{L}$. The results for the tumor markers were as follows: alpha-fetoprotein (AFP), $2.45 \mathrm{ng} / \mathrm{mL}$; protein induced by vitamin $\mathrm{K}$ absence or antagonist-II (PIVKA-II), $18 \mathrm{mAU} / \mathrm{mL}$; carcinoembryonic antigen (CEA), $4.27 \mathrm{ng} / \mathrm{mL}$; carbohydrate antigen 19-9 (CA 19-9), 16.07 U/mL. The results of all blood tests were normal, except for a mild elevation of $\mathrm{LDH}$. The viral serology test revealed hepatitis B surface antigen negativity, hepatitis B surface antibody positivity, hepatitis B core antibody negativity, and hepatitis $\mathrm{C}$ antibody negativity.

\section{Image and pathologic findings}

Multiphase contrast-enhanced CT of the abdomen revealed multinodular masses in both hepatic lobes measuring $6 \mathrm{~cm}$ in the greatest diameter and typical radiological features of HCC, arterial phase hyperenhancement, and washout during the portal venous and delayed phases. However, there was no evidence of cirrhosis (Fig. 1). Multiphase MRI of the liver, performed using a hepatobiliary contrast agent, re- vealed multiple lobulated masses in both hepatic lobes (Fig. 2). The tumor showed hyperenhancement during the arterial phase and washout during the portal venous, delayed, and hepatobiliary phases; these are consistent with the imaging hallmarks of HCC. However, the tumor demonstrated high signal intensity on fat-suppressed T1-weighted images, which was suggestive of hemorrhage or melanin. Ultrasonography (US)-guided percutaneous biopsy of the liver revealed malignant cells consistent with malignant melanoma (Fig. 3). 18F-fluorodeoxyglucose (FDG) positron emission tomography and CT (PET-CT) revealed hypermetabolic lesions in the liver, right upper lobe of the lung, and right pelvic bone (Fig. 4).

\section{Diagnosis and treatment progress}

She was diagnosed with a recurrence of malignant melanoma involving the liver, lung, and bone. Molecular testing of $B R A F$ mutations using polymerase chain reaction confirmed $B R A F$ wild-type melanoma. Finally, treatment with pembrolizumab was initiated. PET-CT after 3 cycles of chemotherapy showed a partial response, and the patient is currently continuing the same treatment.

\section{DISCUSSION}

HCC is the most common primary malignant tumor of the liver, and it usually occurs in patients with chronic liver diseases, such as chronic hepatitis $\mathrm{B}$, chronic hepatitis $\mathrm{C}$, and
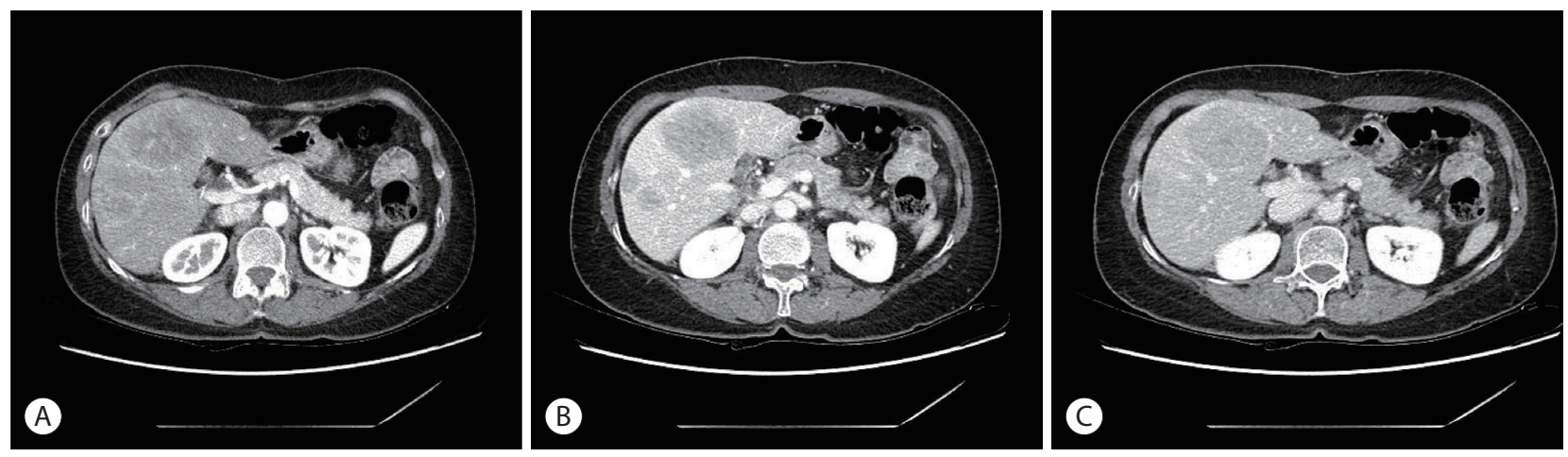

Figure 1. Liver dynamic computed tomography image findings. Multinodular liver masses measuring $6 \mathrm{~cm}$ in the greatest diameter in both hepatic lobes show heterogeneous enhancement during the arterial phase $(A)$ and washout during the portal venous and delayed phases $(B, C)$. 
liver cirrhosis. HCC can be diagnosed either pathologically with biopsy or with noninvasive imaging in patients with risk factors. In clinical practice, HCC is often accompanied by underlying liver cirrhosis, and biopsy is often difficult to perform because of poor liver function (presence of ascites, risk of bleeding), concerns for needle track seeding, and challenges in tumor targeting. Thus, most patients with HCC are diagnosed based on noninvasive imaging findings. In at-risk patients (chronic hepatitis B, chronic hepatitis $\mathrm{C}$, and liver cirrhosis) with nodules larger than or equal to $1 \mathrm{~cm}$, HCC can be diagnosed based on typical imaging hallmarks, including hyperenhancement during the arterial phase and washout during the portal venous and delayed phases on multiphase contrast-enhanced CT or MRI. ${ }^{1}$ A recent metaanalysis of the diagnostic performance of CT and MRI showed sensitivities of $76 \%$ for CT and $83 \%$ for MRI, with specificities of $91 \%$ for CT and $89 \%$ for MRI. ${ }^{5}$ The diagnostic accuracies of CT and MRI vary with the size of HCC, and MRI provides better sensitivity than CT for small HCCs (sensitivity of $48 \%$ and $62 \%$ for CT and MRI in tumors smaller than $20 \mathrm{~mm}$ vs. $92 \%$ and $95 \%$ for CT and MRI in tumors larger than $20 \mathrm{~mm}){ }^{6}$ Therefore, if a patient without clinical risk factors of HCC has inconclusive imaging findings on CT and MRI, a biopsy of the liver mass for pathologic confirmation is necessary.

The liver is one of the most common sites of metastasis. The most frequent primary origins of liver metastases are the lung, colorectal, pancreas, stomach, and breast. ${ }^{7}$ Metastatic liver cancers are usually multiple, and their radiologic findings vary with their origin. Most metastatic liver cancers are hypovascular and show decreased enhancement during the arterial phase, becoming more prominent during the delayed phases. On the other hand, some hypervascular metastases arising from hypervascular primary tumors, such as melanoma, neuroendocrine tumor, renal cell carcinoma, and thyroid carcinoma, may show early enhancement during the arterial phase and washout during the delayed phases.

Signal intensity patterns on unenhanced images of most HCCs generally exhibit hypointensity on T1-weighted images and hyperintensity on T2-weighted images. Some HCCs

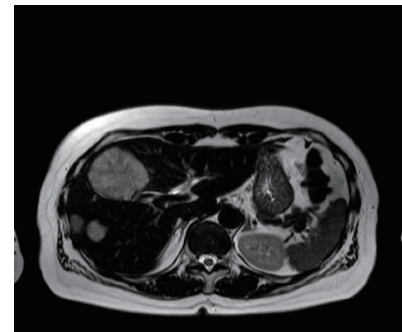

A
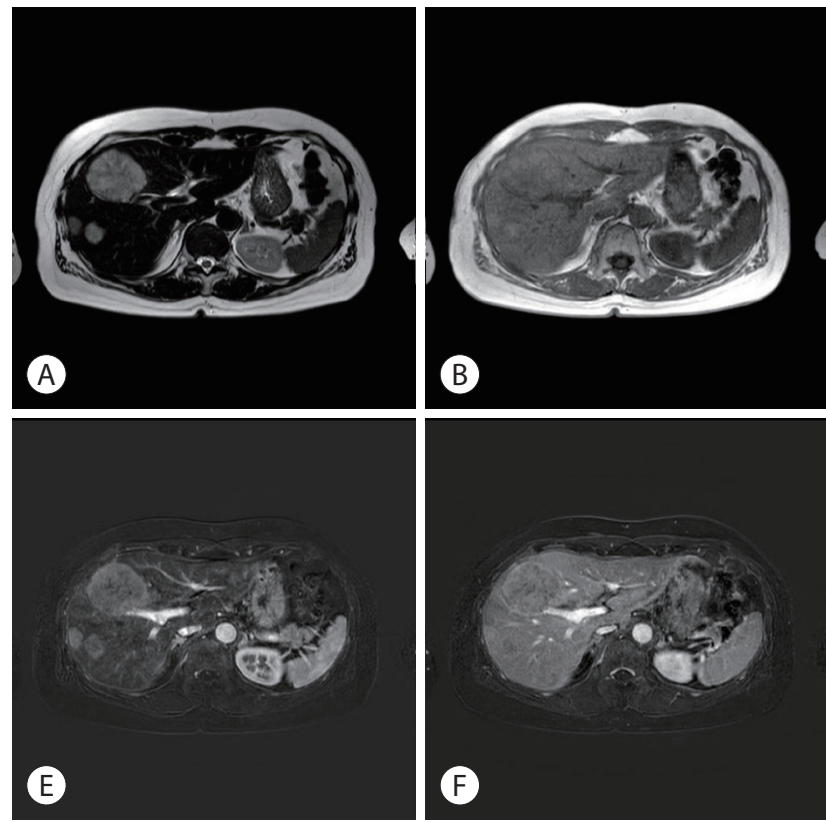
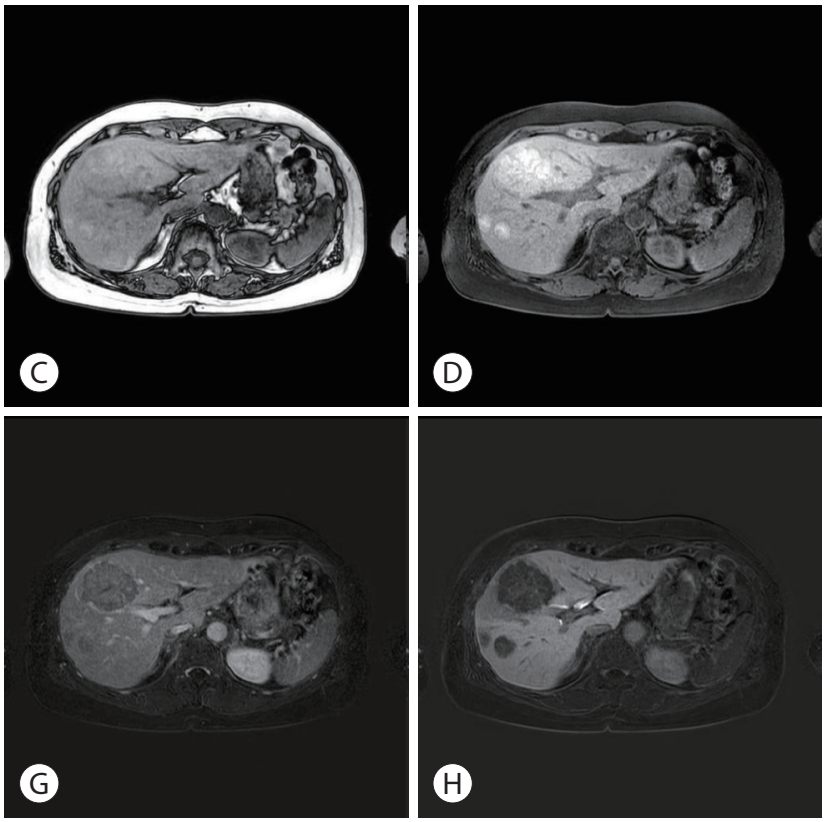

Figure 2. Liver dynamic magnetic resonance imaging image findings. Multiple liver masses show hyperintensity on T2-weighted images (A), isointensity on T1-weighted in-phase and out-of-phase images (B, C). Fat suppression increases the conspicuity of the lesion. A fat-suppressed T1weighted image shows high signal intensity (D), a finding that suggests hemorrhage or melanin. Dynamic images after the administration of contrast agents show hyperintensity during the arterial phase $(\mathrm{E})$, isointensity during the portal venous phase $(\mathrm{F})$, and hypointensity during the delayed phase $(\mathrm{G})$ and hepatobiliary phase $(\mathrm{H})$. 
with fat or hemorrhage may be hyperintense on T1-weighted images. Hypervascular metastases vary but generally exhibit hypointensity on T1-weighted images and hyperintensity on T2-weighted images. However, some metastases that contain hemorrhage, fat, mucin, or melanin may have hyperintensity on T1-weighted images. ${ }^{2-4}$

As mentioned above, the imaging features of hypervascular metastases overlap with those of HCCs. Therefore, clinical factors, such as the absence of chronic liver disease, normal tumor markers, and a history of primary extrahepatic malignancy, suggesting metastatic tumors are more important.

Malignant melanoma is a malignant tumor originating from melanocytes of the skin and other organs with a high potential for metastases and a high mortality rate. It is a rare disease in Asian countries, but its incidence has been increasing in Korea. ${ }^{8}$ Malignant melanoma may involve any organ, and it commonly metastasizes to the lymph nodes, lungs, liver, brain, or bone. ${ }^{9}$ The median overall survival for patients with distant metastases is reported to be 7-8 months. ${ }^{10}$ The current treatments for patients with metastatic or unresectable disease include immunotherapy (such as pembrolizumab, nivolumab, nivolumab, and ipilimumab) and combined targeted therapy (such as vemurafenib with cobimetinib) for BRAF V600-mutated melanoma. ${ }^{11}$

In this report, we present a case of liver metastasis of a malignant melanoma mimicking HCC with a similar enhancement pattern on dynamic contrast imaging. At the time of diagnosis, the patient had no underlying liver disease and was not drinking alcohol. The results of the tumor markers were also normal, so she had no clinical risk factors for HCC. On the other hand, she had a history of malignant melanoma, and the tumor showed high signal intensity on T1weighted images, a characteristic feature of malignant melanoma due to the presence of melanin. We needed to differentiate between HCC and metastatic liver cancer. Therefore, we performed a histological examination using a percutaneous liver biopsy and confirmed the recurrence of

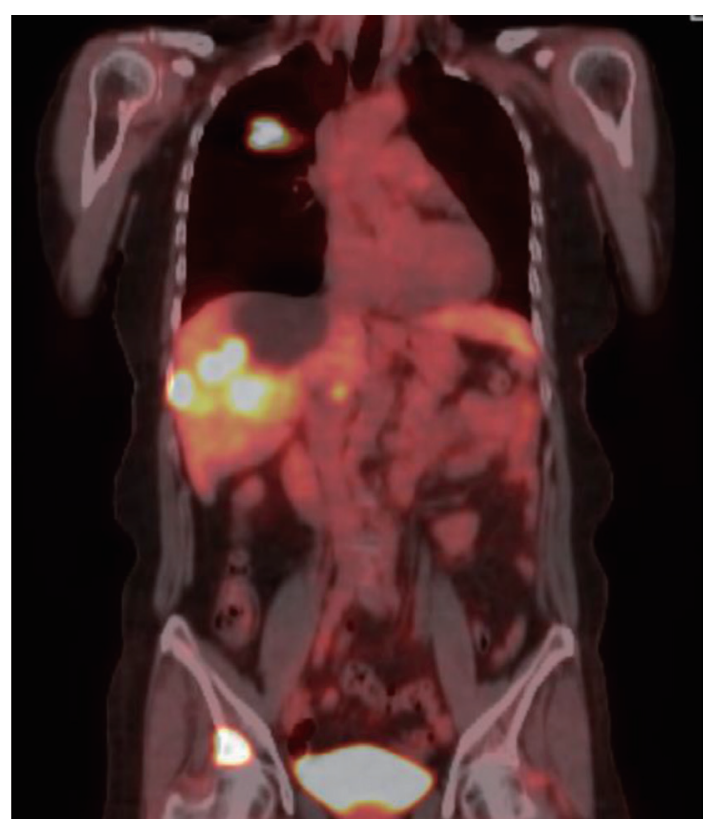

Figure 4. Positron emission tomography and computed tomography findings. There are hypermetabolic lesions in the liver, the right upper lobe of the lung, and the right pelvic bone.
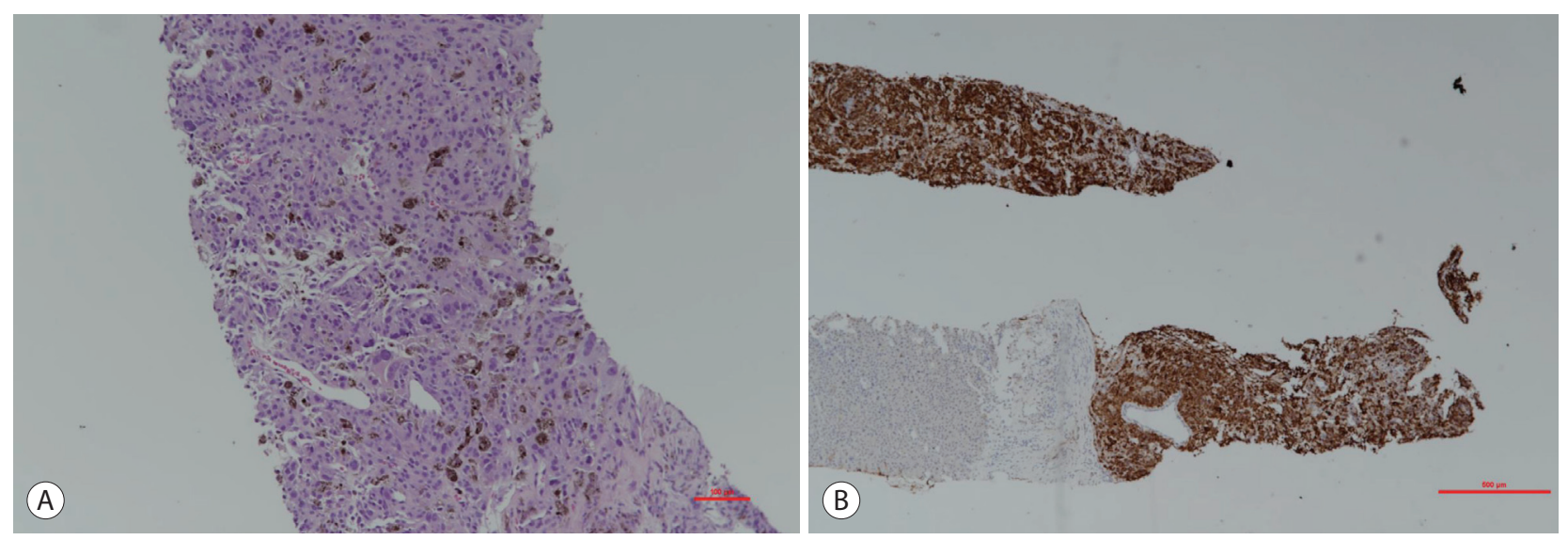

Figure 3. Pathologic findings of the liver biopsy specimen. The tumor cells show brown-pigment cytoplasmic pigmentation, which is consistent with malignant melanoma $(A$, hematoxylin and eosin $\times 100)$. Immunohistochemical staining for Melan- $A$ (melanocyte antigen) is positive $(B, \times 40)$. 
malignant melanoma in the liver.

As in this case, recurrence after a long period is often reported even if the patient achieves complete remission of the primary cancer. Therefore, metastatic liver cancer should be considered as a differential diagnosis if the patient has a history of other primary malignancies. In particular, we need to be cautious of hypervascular cancers that may mimic HCC. Biopsy of the liver mass should be considered for the differential diagnoses of other hypervascular tumors in patients with a history of other primary malignancies without underlying chronic liver disease.

\section{Conflicts of Interest}

The authors have no conflicts of interest to disclose.

\section{REFERENCES}

1. Korean Liver Cancer Association; National Cancer Center. 2018 Korean Liver Cancer Association-National Cancer Center Korea Practice Guidelines for the Management of Hepatocellular Carcinoma. Gut Liver 2019;13:227-299.

2. Ros PR, Erturk SM. Malignant tumors of the liver. In: Gore RM, Levine MS, eds. Textbook of gastrointestinal radiology. Vol 2. 4th ed. Philadephia: Saunders, 2015:1561-1607.

3. Matos AP, Velloni F, Ramalho M, AlObaidy M, Rajapaksha A, Semelka RC. Focal liver lesions: practical magnetic resonance imaging approach. World J Hepatol 2015;7:1987-2008.
4. Kim YY, Park MS, Aljogiman KS, Choi JY, Kim MJ. Gadoxetic acidenhanced magnetic resonance imaging: Hepatocellular carcinoma and mimickers. Clin Mol Hepatol 2019;25:223-233.

5. Chou R, Cuevas C, Fu R, Devine B, Wasson N, Ginsburg A, et al. Imaging techniques for the diagnosis of hepatocellular carcinoma: a systematic review and meta-analysis. Ann Intern Med 2015;162:697-711.

6. Lee YJ, Lee JM, Lee JS, Lee HY, Park BH, Kim YH, et al. Hepatocellular carcinoma: diagnostic performance of multidetector CT and MR imaging-a systematic review and meta-analysis. Radiology 2015;275:97-109.

7. Wang S, Feng Y, Swinnen J, Oyen R, Li Y, Ni Y. Incidence and prognosis of liver metastasis at diagnosis: a pan-cancer populationbased study. Am J Cancer Res 2020;10:1477-1517.

8. Oh CM, Cho H, Won YJ, Kong HJ, Roh YH, Jeong KH, et al. Nationwide trends in the incidence of melanoma and non-melanoma skin cancers from 1999 to 2014 in South Korea. Cancer Res Treat 2018;50:729-737.

9. Patel JK, Didolkar MS, Pickren JW, Moore RH. Metastatic pattern of malignant melanoma. A study of 216 autopsy cases. Am J Surg 1978:135:807-810.

10. Barth A, Wanek LA, Morton DL. Prognostic factors in 1,521 melanoma patients with distant metastases. J Am Coll Surg 1995; 181:193-201

11. NCCN clinical practice guidelines in oncolology. Melanoma: Cutaneous (version 1.2021) [Internet]. Plymouth Meeting, PA: National Comprehensive Cancer Network; [cited 2020 Nov 25]. Available from: https://www.nccn.org/professionals/physician_gls/pdf/cutaneous_melanoma.pdf. 